\title{
Partially integrable systems in multi-dimensions by a variant of the dressing method. 1
}

\author{
A.I. Zenchuk \\ Center of Nonlinear Studies of L.D.Landau Institute for Theoretical Physics \\ (International Institute of Nonlinear Science) \\ Kosygina 2, Moscow, Russia 119334 \\ E-mail: zenchuk@itp.ac.ru \\ P.M. Santini \\ Dipartimento di Fisica, Università di Roma "La Sapienza" \\ and Instituto Nazionale di Fisica Nucleare, Sezione di Roma1, \\ Piazz.le Aldo Moro 2, I-00185 Roma, Italy \\ E-mail: paolo.santini@roma1.infn.it
}

September 30, 2018

\begin{abstract}
In this paper we construct nonlinear partial differential equations in more than 3 independent variables, possessing a manifold of analytic solutions with high, but not full, dimensionality. For this reason we call them "partially integrable". Such a construction is achieved using a suitable modification of the classical dressing scheme, consisting in assuming that the kernel of the basic integral operator of the dressing formalism be nontrivial. This new hypothesis leads to the construction of: 1) a linear system of compatible spectral problems for the solution $U(\lambda ; x)$ of the integral equation in 3 independent variables each (while the usual dressing method generates spectral problems in 1 or 2 dimensions); 2) a system of nonlinear partial differential equations in $n$ dimensions $(n>3)$, possessing a manifold of analytic solutions of dimension $(n-2)$, which includes one largely arbitrary relation among the fields. These nonlinear equations can also contain an arbitrary forcing.
\end{abstract}

\section{Introduction}

Since the discovery of the integrability of the Korteweg-de Vries equation [1], much effort has been devoted to the study of direct techniques to construct and solve nonlinear Partial Differential Equations (PDEs). One of the most powerful of such techniques is the dressing method, originally introduced in [2] and subsequently generalized in [3]-[6] (see also [7, 8]), which is based on the existence of a linear analyticity problem, i.e. a Riemann-Hilbert or a $\bar{\partial}$ problem in some spectral variable $\lambda$ for some matrix function $U(\lambda ; x)$, depending parametrically on the space-time variables $x=\left(x^{1}, \ldots, x^{n}\right)$. (The $\bar{\partial}$ problem was introduced, in the context of 
integrable systems, in $[9,10]$.) Such an analyticity problem is characterized by a linear integral equation, whose unique solvability allows one to construct and solve an overdetermined system of compatible linear spectral problems for $U(\lambda ; x)$, and, consequently, a nonlinear system of PDEs in the independent variables $x$, for the coefficients of such a linear system.

The manifold of the analytic solutions of the nonlinear PDEs constructed by the dressing method is parameterized in terms of a proper number of arbitrary spectral functions, appearing in the linear integral equation, which depend on $n-1$ variables. Therefore the solution space is full and we say that the nonlinear PDE is completely integrable. For instance, the solution space of $1+1$ dimensional scalar systems like the Korteweg-de Vries and the nonlinear Schrödinger equations has dimension 1 , being parameterized by an arbitrary function of 1 variable, while the solution space of the $2+1$ dimensional generalizations of them, the Kadomtsev-Petviashvili and the Davey-Stewartson equations, has dimensionality 2, being parameterized by an arbitrary function of 2 variables.

Motivated by the above considerations, in this paper we say that the dimensionality of the space of analytic solutions of a system of PDEs is $k$, if the analytic solutions are parameterized by a "sufficient number" of arbitrary functions of $k$ independent variables. For instance, if the system of PDEs contains $K$ equations involving first order "time"-derivatives of $K$ functions, then the "sufficient number" is $K$. If $k=n-1$, then the systems is completely integrable.

One of the most outstanding open problems in the theory of integrable systems is the construction of nonlinear PDEs in multi-dimensions (see, for instance, $[12,13]$ ), i.e., in more than 3 dimensions, which could be integrated using suitable extensions of the above dressing procedure. Apart from few exceptional instances, among which one counts the self-dual YangMills equations [11] and the Plebanski heavenly equation [14] (see [15, 16, 17, 18] and [19, 20] for their integration schemes), no significant examples are known in the literature [21].

The purpose of this paper is the construction of PDEs in more than 3 independent variables, possessing a manifold of analytic solutions with high, but not full, dimensionality. For this reason we call such PDEs "partially integrable". This construction is achieved using a suitable modification of the classical dressing scheme, consisting in assuming that the kernel of the basic integral operator of the dressing formalism be nontrivial. As we shall see, this new hypothesis leads to the construction of:

1. a linear system of compatible spectral problems in 3 independent variables each, for the eigenfunction $U(\lambda ; x)$, where $\lambda$ is a vector spectral parameter (while the usual dressing method generates spectral problems in 1 or 2 dimensions with scalar spectral parameter);

2. partially integrable nonlinear PDEs in $n$ dimensions, possessing a solution space of dimension $(n-2)$.

A prototype example is given by the following 4 dimensional system of two matrix equations

$$
\mathcal{B}_{2}\left(q_{1}, q_{1}, q_{2}\right) \mathcal{B}_{2}^{-1}\left(q_{1}, q_{2}, q_{3}\right)=\mathcal{B}_{3}\left(q_{1}, q_{1}, q_{2}\right) \mathcal{B}_{3}^{-1}\left(q_{1}, q_{2}, q_{3}\right)=\mathcal{B}_{4}\left(q_{1}, q_{1}, q_{2}\right) \mathcal{B}_{4}^{-1}\left(q_{1}, q_{2}, q_{3}\right)
$$

for the three square matrix fields $q_{1}(x), q_{2}(x), q_{3}(x)$, supplemented by the "largely arbitrary" relation

$$
F\left(q_{1}, q_{2}, q_{3}\right)=0
$$

among them, where the matrix blocks $\mathcal{B}_{j}$ are defined as:

$$
\mathcal{B}_{j}\left(q_{1}, q_{2}, q_{3}\right) \equiv q_{2 x^{j}}-q_{2 x^{1}} B_{j}-q_{2}\left[q_{1}, B_{j}\right]-\left[B_{j}, q_{3}\right], \quad j=2,3,4,
$$


$B_{j}, j=2,3,4$ are constant diagonal matrices different from the identity, and $[\cdot, \cdot]$ is the usual commutator of matrices. In the simplest case, the largely arbitrary relation (2) can be chosen to be an equation defining one of the fields, say $q_{3}$, to be any given function $\gamma(x)$ (in general, a generalized function), interpretable as an "external arbitrary forcing":

$$
F: \quad q_{3}(x)=\gamma(x)
$$

As we shall see in the following, equations (1-4) possess a manifold of analytic solutions of dimension 2.

The above closed system of equations (1-2) share with the other models constructed in this paper the following properties.

1. The existence of a nontrivial kernel of the basic integral equation implies that the solutions constructed by the dressing depend on an arbitrary function $f(x)$ of the coordinates; this fact has the following important implications.

2. The nonlinear system of PDEs constructed by the dressing scheme possesses a distinguished block structure (see (1)) and is under-determined.

3. To close the system and fix its indeterminacy (or, equivalently, to fix $f(x)$ ), one has to introduce an "external and largely arbitrary" relation among the fields (see (2)). If, for instance, such a relation (algebraic or differential) is linear, then the construction of explicit solutions via the dressing algorithm remains linear as well. The simplest example of linear relation is obtained imposing that one of the fields be a given function of the coordinates, like in (4), interpretable as an external forcing.

4. The system of PDEs depends on two types of matrix fields, those obtained "saturating the vector parameter $\lambda$ " of the solution $U(\lambda ; x)$ of the linear integral equation by ingredients of the classical dressing method, whose dimensionality is constrained, and those obtained saturating $\lambda$ by a novel dressing function $G(\lambda ; x)$, whose dimensionality is not constrained. That's why the dimensionality of the solution space, $(n-2)$, can be arbitrarily large.

5. While integrable PDEs in low dimensions $(2+1$ or less $)$ are the compatibility of overdetermined systems of linear spectral problems, such a feature seems to be lost for our higher dimensional examples.

We remark that partially integrable equations of the type (1) are somehow connected to the $\mathrm{N}$-wave type systems; indeed the two equations

$$
\mathcal{B}_{2}\left(q_{1}, q_{1}, q_{2}\right)=\mathcal{B}_{3}\left(q_{1}, q_{1}, q_{2}\right)=0
$$

are equivalent to the $\mathrm{N}$-wave system in $2+1$ dimensions for the field $q_{1}$, obtained eliminating $q_{2}$ from equations (5) (see also Sec. 2.1). The construction of partially integrable PDEs connected to other basic integrable systems, like those belonging to the Kadomtsev-Petviashvili hierarchy, or those associated with the Davey-Stewartson equation, will be the subject of a forthcoming paper.

We also remark that a different generalization of the dressing procedure, allowing to construct a class of partially integrable PDEs which combine $S$ and $C$ integrability features, has been already proposed in [22] (a nonlinear system is $S$ integrable, like the KdV equation, if it is solved via a linear integral equation; it is $C$ integrable, like the Burgers equation, if it 
is linearized by a simpler change of variables, like a contact transformation; see [23] for more details on these definitions).

The paper is organized as follows. In Sec. 2 we derive, for the sake of comparison, the well-known $N$-wave system in (2+1)-dimensions, using the classical dressing method, and we show that its solution space is full. Then in Sec. 3 we explore the implications of the existence of a nontrivial kernel of the basic integral operator of the dressing scheme, and we construct examples of nonlinear $n$-dimensional PDEs possessing a space of analytic solutions of dimension $n-2$. We also show that these equations do not seem to be the commutativity condition of overdetermined systems of linear PDEs. In Sec. 4 we show how to construct an integral operator possessing a nontrivial kernel, and we use this result to characterize a large class of analytic solutions of the partially integrable PDEs of this paper. A natural extension of the algorithm presented in this paper is briefly mentioned in the final Sec. 5.

\section{Derivation of classical integrable systems using the dressing algorithm}

The starting point of the dressing constructions contained in this paper is the linear integral equation

$$
\Phi(\lambda ; x)=\int \Psi(\lambda, \mu ; x) U(\mu ; x) d \Omega(\mu) \equiv \hat{\Psi} U(\lambda ; x),
$$

in the spectral variables $\lambda, \mu$, for the unknown matrix function $U$. The given matrix functions $\Phi$ and $\Psi$ are defined by some extra conditions, which fix their dependence on an additional vector parameter $x=\left(x^{1}, \ldots, x^{n}\right)$, whose components are the independent variables of the associated nonlinear PDEs. $\Omega$ is some largely arbitrary scalar measure in the $\mu$-space. Apart from $\Omega$, all the functions appearing in this paper are $Q \times Q$ matrix functions.

We remark that no a priori assumption is made in (6) on the dependence of $\Psi$ on $\lambda$ (this general starting point has been used, for instance, in [24] and in [22]), to keep the structure of $\Psi$ as much general as possible. Indeed, although in most of the cases such a dependence is described by a Cauchy kernel, an indication that equation (6) is the manifestation of RiemannHilbert and/or $\bar{\partial}$ analyticity problems, there are examples (see [24] and [22]) in which more general representations appear, indicating that the above analyticity problems could be a too restrictive starting points.

Before developing, in Sec. 3, 4, the novel features of the dressing method, it is useful to summarize the essential steps of the classical dressing method used to construct and solve the classical 3-dimensional $N$-wave system (18) (which is known to be an $S$-integrable system), together with its solution space. Such a solution space is 2 dimensional (i.e., it is complete), being parameterized by an arbitrary function of 2 variables.

\subsection{S-integrable PDEs: the $N$-wave system.}

The basic assumption underlying all the known dressing procedures available in the literature, is that the operator $\hat{\Psi}$ in (6) be uniquely invertible; i.e., that

$$
\operatorname{dim} \operatorname{ker} \hat{\Psi}=0 \text {. }
$$


The $x$-dependence is introduced by the matrix equations

$$
\Psi_{x^{i}}(\lambda, \mu ; x)=\Phi(\lambda ; x) B_{i} C(\mu ; x), \quad i=1, . ., \operatorname{dim} x,
$$

showing that the $x$-derivatives of the kernel $\Psi$ are degenerate matrix functions of the spectral parameters, another basic feature of all known dressing algorithms, where $B_{i}, i=1, . ., n$, are constant diagonal matrices, so at most $Q$ of them may be independent. Due to the above degeneracy, the compatibility of equations (8) leads to separate equations for $\Phi$ and $C$ :

$$
\begin{gathered}
\Phi_{x^{i}} B_{j}-\Phi_{x^{j}} B_{i}=0, \quad i \neq j, \\
B_{j} C_{x^{i}}-B_{i} C_{x^{j}}=0, \quad i \neq j,
\end{gathered}
$$

and one equation is the adjoint of the other. Without loss of generality we assume $B_{1}=I$, where $I$ is the identity matrix.

Replacing, in equation (9), $\Phi$ by $\hat{\Psi} U$, as indicated in (6), and using (8), one obtains the following equation:

$$
\hat{\Psi} L_{i j} U=0
$$

where

$$
L_{i j} U \equiv U_{x^{i}} B_{j}-U_{x^{j}} B_{i}+U B_{i} v B_{j}-U B_{j} v B_{i}, \quad i, j=1, . ., \operatorname{dim} x, \quad i \neq j
$$

and

$$
v(x) \equiv \int C(\lambda ; x) U(\lambda ; x) d \Omega(\lambda)
$$

Then the property (7) implies that

$$
L_{i j} U(\lambda ; x)=0, \quad i, j=1, . ., \operatorname{dim} x, \quad i \neq j
$$

or, explicitly:

$$
\begin{aligned}
& L_{21} U=U_{x^{2}}-U_{x^{1}} B_{2}-U\left[v, B_{2}\right]=0, \\
& L_{31} U=U_{x^{3}}-U_{x^{1}} B_{3}-U\left[v, B_{3}\right]=0,
\end{aligned}
$$

having chosen $j=1, i=2,3$.

This is nothing but the well-known linear overdetermined system corresponding to the $\mathrm{N}$ wave equation in the three variables $x^{1}, x^{2}, x^{3}$.

The associated complete system of nonlinear PDEs is simply obtained, in the dressing philosophy, upon "saturating the parameter $\lambda$ " in equations (15) by the integral operator $\int d \Omega(\lambda) C(\lambda ; x) \cdot:$

$$
\begin{gathered}
L_{21} v-\left[B_{2}, v^{1}\right]=v_{x^{2}}-v_{x^{1}} B_{2}-v\left[v, B_{2}\right]-\left[B_{2}, v^{1}\right]=0, \\
L_{31} v-\left[B_{3}, v^{1}\right]=v_{x^{3}}-v_{x^{1}} B_{3}-v\left[v, B_{3}\right]-\left[B_{3}, v^{1}\right]=0 .
\end{gathered}
$$

It is written in terms of the square matrix fields $v(x)$ and $v^{1}(x)$, where

$$
v^{1}(x) \equiv \int C_{x^{1}}(\lambda ; x) U(\lambda ; x) d \Omega(\lambda)
$$


Eliminating $v^{1}$ from these two equations, we get the celebrated $N$-wave system in 3 dimensions:

$$
\left[v_{x^{3}}, B_{2}\right]-\left[v_{x^{2}}, B_{3}\right]+B_{2} v_{x^{1}} B_{3}-B_{3} v_{x^{1}} B_{2}-\left[\left[v, B_{2}\right],\left[v, B_{3}\right]\right]=0
$$

The same equation may be derived directly from the compatibility condition of the system (15).

Similarly, considering the equations $L_{j 1} U=0$ and $L_{k 1} U=0$ for any $j \neq k \neq 1$, one derives the hierarchy of $n$-wave equations

$$
\left[v_{x^{k}}, B_{j}\right]-\left[v_{x^{j}}, B_{k}\right]+B_{j} v_{x^{1}} B_{k}-B_{k} v_{x^{1}} B_{j}-\left[\left[v, B_{j}\right],\left[v, B_{k}\right]\right]=0
$$

We remark that, in the above dressing construction, the linear integral operator $\hat{\Psi}$ in (6) acts from the left and, consequently, the partial differential operators $L_{i j}$ in (14) act from the right, while, in the soliton literature, one usually makes the opposite choice.

Our choice is motivated by the fact that, as we shall see in Sec.3,4, in more than $2+1$ dimensions, the role played by the linear integral equation (6) seems to be more fundamental than that played by the linear overdetermined system of PDEs. Indeed, while integrable PDEs in $2+1$ dimensions (or less) are characterized as the compatibility condition of a linear overdetermined system of PDEs, such a basic property seems to be lost in multi-dimensions. Instead, as we shall see in the following sections, the linear integral equation (6) can generate nonlinear PDEs, together with their large manifold of analytic solutions, also in the multidimensional context.

\subsection{Solution space}

We now consider the manifold of particular solutions of equations $(18,19)$. The solutions of eqs.(9) and (10) can be parameterized as follows:

$$
\begin{aligned}
& \Phi(\lambda ; x)=\int \Phi_{0}(\lambda, k) e^{k B \cdot x} d k \\
& C(\mu ; x)=\int e^{q B \cdot x} C_{0}(\mu, q) d q
\end{aligned}
$$

where

$$
B \cdot x=\sum_{i=1}^{n} B_{i} x^{i}
$$

and where the spectral parameters $\lambda, \mu, k, q$ are necessarily scalars. Thus equations (8) yield:

$$
\Psi(\lambda, \mu ; x)=\int \Phi_{0}(\lambda, k) e^{(k+q) B \cdot x} C_{0}(\mu, q) \frac{d k d q}{k+q}+\Sigma(\lambda, \mu), \quad B_{1}=I
$$

where the integration constant $\Sigma(\lambda, \mu)$ is chosen here to be $\delta(\lambda-\mu)$.

It is simple to see, from the linear limit, that the solution space of equation (18), generated by the dressing algorithm, is full. Indeed, in the linear limit: $\Psi(\lambda, \mu) \sim \delta(\lambda-\mu)$ and $U \sim \Phi$. Take $C_{0}(\lambda, q)=\delta(\lambda-q)$; then the solution $v$ of the 3 - dimensional $N$-wave system (18), which in the linear limit reads

$$
v(x) \sim \int C(\lambda ; x) \Phi(\lambda ; x) d \Omega(\lambda)=\int e^{\lambda B \cdot x} \Phi_{0}(\lambda, k) e^{k B \cdot x} d k d \Omega(\lambda),
$$


is parameterized by the arbitrary matrix function $\Phi_{0}(\lambda, k)$ of the two scalar spectral parameters $\lambda, k$; then its solution space is 2 dimensional, and therefore it is complete.

We end this section remarking that the Cauchy kernel appearing in (23), obtained here as a consequence of equations $(20),(21)$ and (8), is a manifestation of the distinguished analyticity properties of the solution $U(\lambda ; x)$ in the complex $\lambda$ plane, in agreement with the well-known derivations of the $N$-wave equation (18) from Riemann-Hilbert and /or $\bar{\partial}$ problems [25, 26, 27].

\section{Partially integrable PDEs in multi-dimensions.}

In this section we show how to construct partially integrable PDEs in $n$ dimensions exhibiting a space of analytic solutions of dimension $(n-2)$.

\subsection{Generalization of the dressing algorithm}

In the Sec.2.1 we have constructed, from the general hypothesis (6), (7) and (8) underlying the classical dressing algorithm, the integrable $N$-wave system in 3 dimensions. The main obstacle to go to higher dimensions is clearly due to the fact that each linear problem $L_{i j} U=0$, as a consequence of $(7)$, is 2 dimensional.

To increase the dimensionality of the linear problems, we then suppose that the kernel of the operator $\hat{\Psi}$ is one dimensional:

$$
\operatorname{dim} \operatorname{ker} \hat{\Psi}=1
$$

i.e., the solution of the homogeneous equation associated with eq.(6) is nontrivial:

$$
0=\hat{\Psi} H \quad \Leftrightarrow \quad H(\lambda ; x)=U^{h}(\lambda ; x) f(x)
$$

where $U^{h}(\lambda ; x)$ is some nontrivial solution of the homogeneous equation $\hat{\Psi} H=0, f(x)$ is an arbitrary matrix function of $x$, and $\lambda, \mu, \nu$ are now vector spectral parameters whose dimension is specified in Sec.4. Then the general solution of eq.(6) reads

$$
U(\lambda ; x)=U^{p}(\lambda ; x)+U^{h}(\lambda ; x) f(x),
$$

where $U^{p}(\lambda ; x)$ is some particular solution of $(6)$.

As a consequence of the novel assumption (25), equation (11) implies the following equations for $U$ :

$$
L_{i j} U(\lambda ; x)=\left(L_{n m} U(\lambda ; x)\right) A^{i j n m}(x), \quad i \neq j, \quad n \neq m
$$

where $A^{i j n m}(x)$ are some matrix functions of $x$ to be specified, reflecting the fact that two solutions of the homogeneous equation $\hat{\Psi} H=0$ differ by a matrix function of $x$, multiplied from right.

Since the following cyclic permutation formula among three operators $L_{i j}$ holds:

$$
\sum_{\operatorname{cycl}(i j k)}\left(L_{i j} \mathcal{M}\right) B_{k}=0, \quad i \neq j \neq k
$$


where $\mathcal{M}$ is an arbitrary square matrix, it follows that only $(n-1)$ operators $L_{i j}$ are linearly independent. Therefore we take the operators $\left\{L_{j 1}, j=2, \ldots, n\right\}$ as elements of the basis, and we consider the following subset of equations (28), involving only these elements:

$$
\begin{aligned}
\mathcal{E}_{j}(\lambda ; x) \equiv & L_{j 1} U(\lambda ; x)-\left(L_{21} U(\lambda ; x)\right) A^{j}(x)=0, \quad j=3, \ldots, n, \\
& L_{j 1} U \equiv U_{x^{j}}-U_{x^{1}} B_{j}-U\left[v, B_{j}\right], \quad j=2, \ldots, \operatorname{dim} x,
\end{aligned}
$$

where $A^{j}(x)$ are some matrix functions to be defined.

We have established that, if $\operatorname{dim} \operatorname{ker} \hat{\Psi}=1$, then each linear equation (30) for the spectral function $U(\lambda ; x)$ is 3 dimensional.

The associated nonlinear equations, obtained "saturating the parameter $\lambda$ " in equations (30) by the integral operator $\int d \Omega(\lambda) C(\lambda ; x) \cdot$, read

$$
L_{j 1} v-\left[B_{j}, v_{1}\right]-\left(L_{21} v-\left[B_{2}, v^{1}\right]\right) A^{j}=0, \quad j=3, . ., n
$$

they are given in terms of the fields $v(x)$ and $v^{1}(x)$, defined respectively in (13) and (17), and of the matrices $A^{j}(x)$. More explicitly, one obtains:

$$
v_{x^{j}}-v_{x^{1}} B_{j}-v\left[v, B_{j}\right]-\left[B_{j}, v^{1}\right]-\left(v_{x^{2}}-v_{x^{1}} B_{2}-v\left[v, B_{2}\right]-\left[B_{2}, v^{1}\right]\right) A^{j}=0, \quad j=3, . ., n .
$$

In order to express $A^{j}(x)$ in terms of $U$ and close the system, we introduce an external dressing function $G(\lambda ; x)$, and the associated new matrix fields

$$
\begin{array}{r}
w^{00}(x) \equiv \int G(\lambda ; x) U(\lambda ; x) d \Omega(\lambda), w^{j 0}(x) \equiv \int G_{x^{j}}(\lambda ; x) U(\lambda ; x) d \Omega(\lambda), \quad j>0, \\
w^{i j}(x) \equiv \int G_{x^{i} x^{j}}(\lambda ; x) U(\lambda ; x) d \Omega(\lambda), \quad i, j>0, \quad w^{i j}(x)=w^{j i}(x) .
\end{array}
$$

The equations for the fields $w^{i j}$ can be derived applying $\int d \Omega(\lambda) G(\lambda) \cdot$ and $\int d \Omega(\lambda) G_{x^{n}}(\lambda)$. to the linear equation (30), obtaining:

$$
L_{j 1} w^{n 0}-w^{j n}+w^{1 n} B_{j}=\left(L_{21} w^{n 0}-w^{2 n}+w^{1 n} B_{2}\right) A^{j}, j=3, . ., \operatorname{dim} x, n=0,1, . ., \operatorname{dim} x .
$$

Some of these equations can be taken as definition of $A^{j}$. But, to close the system, one needs to introduce the following additional structures.

(a) Equations defining $G(\lambda ; x)$. Since $G$ is an outer dressing function, these equations can be a quite arbitrary system of compatible and solvable partial differential equations, either linear or nonlinear, involving derivatives of any order and dimension. This freedom plays a key role in allowing for a large solution space.

(b) An additional relation between all the matrix fields, which may be taken in quite arbitrary form

$$
F\left(v, v^{1}, w^{00}, w^{i 0}, w^{i j}\right)=0, \quad i, j=1,2, \ldots
$$

This equation is needed to fix the arbitrary function $f(x)$ of the variables $x^{i}$ appearing in the solution of the inhomogeneous equation (6) (see equations $(26,27)$ ). The relation (37) is largely arbitrary; the only requirement is that it should give rise to a solvable equation for $f(x)$. Since 
$U$ depends linearly on the arbitrary function $f(x)$, all the fields depend linearly on $f$ as well. Indeed, using equations $(13,17,34)$ and equation $(27)$, one obtains

$$
\begin{aligned}
v(x) & =h_{0}^{v}(x)+h_{1}^{v}(x) f(x), \quad h_{0}^{v}\left(x=\int C(\lambda) U^{p}(\lambda) d \Omega(\lambda), \quad h_{1}^{v}=\int C(\lambda) U^{h}(\lambda) d \Omega(\lambda),\right. \\
v^{1}(x) & =h_{0}^{v^{1}}(x)+h_{1}^{v^{1}}(x) f(x), \quad h_{0}^{v^{1}}=\int C_{x^{1}}(\lambda) U^{p}(\lambda) d \Omega(\lambda), \quad h_{1}^{v^{1}}=\int C_{x^{1}}(\lambda) U^{h}(\lambda) d \Omega(\lambda), \\
w^{00}(x) & =h_{0}^{w^{00}}(x)+h_{1}^{w^{00}}(x) f(x), \quad h_{0}^{w^{00}}=\int G(\lambda) U^{p}(\lambda) d \Omega(\lambda), \quad h_{1}^{w^{00}}=\int G(\lambda) U^{h}(\lambda) d \Omega(\lambda), \\
w^{i 0}(x) & =h_{0}^{w^{i 0}}(x)+h_{1}^{w^{i 0}}(x) f(x), \quad h_{0}^{w^{i 0}}=\int G_{x^{i}}(\lambda) U^{p}(\lambda) d \Omega(\lambda), \quad h_{1}^{w^{i 0}}=\int G_{x^{i}}(\lambda) U^{h}(\lambda) d \Omega(\lambda), \\
w^{i j}(x) & =h_{0}^{w^{i j}}(x)+h_{1}^{w^{i j}}(x) f(x), \quad h_{0}^{w^{i j}}=\int G_{x^{i} x^{j}}(\lambda) U^{p}(\lambda) d \Omega(\lambda), \quad h_{1}^{w^{i j}}=\int G_{x^{i} x^{j}}(\lambda) U^{h}(\lambda) d \Omega(\lambda),
\end{aligned}
$$

where all the $h$ 's are known functions of $x$, and $i, j=1,2, \ldots$ Using this fact, the following types of relation (37) open different scenarios:

1. $F$ is an algebraic expression of its arguments, leading to an algebraic equation for $f$. The simplest case is, of course, that of a linear equation, leading to a linear algebraic equation for $f(x)$. The simplest example of linear algebraic relation is obtained imposing that one of the fields be a "given function of the coordinates, interpretable as an external forcing".

2. $F$ is a multidimensional linear partial differential equation of any dimension and order, either with constant or variable coefficients. This leads to a linear PDE for $f(x)$ having the same dimension and order, and always variable coefficients (due to the functions $h$ in equations (38)).

3. $F$ is a nonlinear PDE, whose dimension $m$ is lower than the dimensionality $n$ of the system of PDEs. This leads to a nonlinear PDE for $f(x)$ in $m$ dimensions, but with variable coefficients. In this case, the dressing procedure allows one to replace the nonlinear PDEs under investigation by a nonlinear PDE for $f(x)$ in lower dimensions (a "reduction of complications").

Among all these cases, the most remarkable one is when $F$ is a linear algebraic relation, since $f(x)$ can be found explicitly and the solution manifold may be constructed analytically (see Sec.4.3 for more details on this point).

The equation defining $G(\lambda ; x)$, together with the relation (37) among the fields, provides the completeness of the nonlinear system of PDEs for the fields $v, v^{1}$ and $w^{i j}$ generated by the dressing.

\subsection{Examples}

In this section we consider some basic examples of partially integrable PDEs, corresponding to special definitions of $G(\lambda ; x)$, and to particular relations (37).

\subsubsection{The simplest nonlinear partially integrable PDEs}

The simplest possible case corresponds to a function $G$ independent of $x$. Then we have the only additional field $w^{00}$, since $w^{i j}=0, i, j>0$. We consider two examples of relation (37). 
1. The relation (37) is chosen as follows:

$$
F: \quad w^{00}(x)=\exp \left[\sum_{i=1}^{n} a_{i} x^{i}\right]
$$

where $a_{j}$ are constant diagonal matrices. Then equation (36) with $n=0$ yields:

$$
A^{j}=\left(a_{2}-a_{1} B_{2}+\left[B_{2}, v\right]\right)^{-1}\left(a_{j}-a_{1} B_{j}+\left[B_{j}, v\right]\right) .
$$

Consequently, equations (33), for each particular choice of $j$, involve just the two fields $v$ and $v^{1}$. Thus we need two equations of this type to close the system, say $j=3,4$, obtaining the following system of 2 matrix equations in 4 dimensions for the matrix fields $v, v^{1}$ :

$$
\begin{aligned}
& \left(L_{21} v-\left[B_{2}, v_{1}\right]\right)\left(a_{2}-a_{1} B_{2}+\left[B_{2}, v\right]\right)^{-1}= \\
& \left(L_{31} v-\left[B_{3}, v_{1}\right]\right)\left(a_{3}-a_{1} B_{3}+\left[B_{3}, v\right]\right)^{-1}=\left(L_{41} v-\left[B_{4}, v_{1}\right]\right)\left(a_{4}-a_{1} B_{4}+\left[B_{4}, v\right]\right)^{-1} .
\end{aligned}
$$

2. The relation (37) is chosen as follows:

$$
F: \quad v^{1}(x)=\gamma(x),
$$

where $\gamma(x)$ is an arbitrary matrix function of $x$. In this case we choose:

$$
A^{j}(x)=\left(L_{21} w^{00}(x)\right)^{-1} L_{j 1} w^{00}(x), \quad j=3,4,
$$

and we obtain the following system of 2 matrix equations in 4 dimensions for the two matrix fields $v, w^{00}$ :

$$
\begin{aligned}
& \left(L_{21} v(x)-\gamma_{2}(x)\right)\left(L_{21} w^{00}(x)\right)^{-1}=\left(L_{31} v(x)-\gamma_{3}(x)\right)\left(L_{31} w^{00}(x)\right)^{-1}= \\
& \left(L_{41} v(x)-\gamma_{4}(x)\right)\left(L_{41} w^{00}(x)\right)^{-1}, \\
& \gamma_{j}(x) \equiv\left[B_{j}, \gamma(x)\right], \quad j=2,3,4,
\end{aligned}
$$

depending on the arbitrary forcing $\gamma(x)$.

In section Sec. 4 we will see that the space of analytic solutions of these two systems is 2-dimensional.

\subsubsection{Partially integrable n-dimensional PDEs}

In this section we first derive nonlinear PDEs in 4 dimensions, and then we turn to nonlinear PDEs in arbitrary dimensions.

Let $G$ be defined by the following equations

$$
G_{x^{j}}=\alpha_{j} G_{x^{1}}, \quad j=1, \ldots, n,
$$

where $\alpha_{j}$ are constant diagonal matrices.

The special form (45) of $G$ implies that the fields $w^{j 0}(x), w^{j n}(x)$ can be expressed in terms of the fields $w^{10}(x), w^{11}(x)$ respectively:

$$
w^{j 0}(x)=\alpha_{j} w^{10}(x), \quad w^{j k}(x)=\alpha_{j} \alpha_{k} w^{11}(x) \quad j, k=1, \ldots, n .
$$


Then all the equations (33) and (36) reduce to the 6 equations (for $j=3,4$ ):

$$
\begin{aligned}
& L_{j 1} v-\left[B_{j}, v^{1}\right]=\left(L_{21} v-\left[B_{2}, v^{1}\right]\right) A^{j}, \\
& L_{j 1} w^{00}-\alpha_{j} w^{10}+w^{10} B_{j}=\left(L_{21} w^{00}-\alpha_{2} w^{10}+w^{10} B_{2}\right) A^{j}, \\
& L_{j 1} w^{10}-\alpha_{j} w^{11}+w^{11} B_{j}=\left(L_{21} w^{10}-\alpha_{2} w^{11}+w^{11} B_{2}\right) A^{j},
\end{aligned}
$$

Equations (49) can be viewed, for instance, as defining the matrix fields $A^{j}, j=3,4$; substituting these definitions of $A^{j}$ in equations (47) and (48), we obtain 4 matrix equations for the 5 matrix fields $v, v^{1}, w^{00}, w^{10}, w^{11}$. Before performing such operations, it is convenient to introduce a more compact notation defining the matrices

$$
E_{j}^{v}:=L_{j 1}(v)-\left[B_{j}, v^{1}\right], \quad E_{j}^{w^{k 0}}:=L_{j 1}\left(w^{k 0}\right)-\alpha_{j} w^{1 k}+w^{1 k} B_{j}, \quad j=3,4, \quad k=0,1 .
$$

Then equation (49) yields

$$
A^{j}=\left(E_{2}^{w^{10}}\right)^{-1} E_{j}^{w^{10}}, j=3,4
$$

and the nonlinear system $(47,48)$ takes the form

$$
\begin{aligned}
E_{j}^{v}\left(E_{j}^{w^{10}}\right)^{-1} & =E_{2}^{v}\left(E_{2}^{w^{10}}\right)^{-1}, \quad j=3,4, \\
E_{j}^{w^{00}}\left(E_{j}^{w^{10}}\right)^{-1} & =E_{2}^{w^{00}}\left(E_{2}^{w^{10}}\right)^{-1}, \quad j=3,4 .
\end{aligned}
$$

This 4 dimensional system is not closed, consisting of 4 equations for the 5 functions $v, v^{1}, w^{00}, w^{10}, w^{11}$. This indeterminacy is consistent with the fact that the above equations are generated by the linear integral equation (6), which possesses a 1 dimensional space of homogeneous solutions. Therefore all the solutions of equations (52-53), constructed by the dressing procedure, contain an arbitrary function $f(x)$.

To fix this arbitrary function, we use the relation (37) which, for this example, reads:

$$
F\left(v, v^{1}, w^{00}, w^{10}, w^{11}\right)=0 .
$$

As we discussed above, this relation is largely arbitrary; the only requirement is that it should give rise to a solvable equation for the function $f(x)$ (see Sec.4.3 for more details on this point).

We remark that the arbitrary relation $(37)$ can be chosen in order to put equations $(52,53)$ into a differential polynomial form. If we choose, for instance, the bilinear relation

$$
F: \quad E_{2}^{w^{10}}=L_{21}\left(w^{10}\right)-\alpha_{2} w^{11}+w^{11} B_{2}=T,
$$

where $T$ is a constant matrix, multiplying equations $(52,53)$ from right by $E_{j}^{w^{10}}$, one transforms them into a differential polynomial form. In addition, if $B^{2}=0$, the bilinear relation becomes linear: $w_{x^{2}}^{10}-\alpha_{2} w^{11}=T$.

We have established that, due to the novel hypothesis $\operatorname{dim} \operatorname{ker} \hat{\Psi}=1$, the dressing algorithm allows one to construct a system of partially integrable PDEs in multi-dimensions which includes one largely arbitrary relation among the fields. This is a novel and surprising feature of the theory.

It is interesting to remark that equations (52-53) admit the reduction

$$
v=w^{00}, v^{1}=w^{10}
$$


which follows imposing that $G(\lambda ; x)=C(\lambda ; x)$, as a consequence of the choice $\alpha_{j}=B_{j}$. In this case, equations (52-53) reduce to the following two nonlinear PDEs

$$
E_{2}^{w^{00}}\left(E_{2}^{w^{10}}\right)^{-1}=E_{3}^{w^{00}}\left(E_{3}^{w^{10}}\right)^{-1}=E_{4}^{w^{00}}\left(E_{4}^{w^{10}}\right)^{-1}
$$

for the three matrix fields $w^{00}, w^{10}, w^{11}$, supplemented by the (largely) arbitrary relation

$$
F\left(w^{00}, w^{10}, w^{11}\right)=0 .
$$

A simple example of linear relation (58) is, for instance,

$$
F: \quad w^{11}(x)=\gamma(x)
$$

where $\gamma$ is an arbitrary function; then the nonlinear system is given by the two equations (57) for the two fields $w^{00}, w^{10}$, with the arbitrary forcing $\gamma(x)$ (it is the system (1-4) presented in the introduction with a different notation).

As we shall see in section 4.4, the above nonlinear PDEs in 4 dimensions possess an analytic solution space of dimension 2 .

One can generalize the above construction, to generate partially integrable PDEs in $n$ dimensions $(n \geq 4)$, whose manifold of analytic solutions has dimension $(n-2)$. This higher dimensional generalization is associated with a more general equation for $G$.

For instance, in order to derive partially integrable PDEs in 5 dimensions, one chooses $G(\lambda ; x)$ to be defined by the following equations:

$$
G_{x^{j}}=\sum_{k=1}^{2} \alpha_{j k} G_{x^{k}}, \quad j>2,
$$

where $\alpha_{j k}$ are constant diagonal matrices. This special form implies that the fields $w^{j 0}, w^{j 1}, w^{j 2}, j>$ 2 can be expressed in terms of the fields $w^{i 0}, w^{i k}, i, k=1,2$ :

$$
w^{j 0}=\sum_{s=1}^{2} \alpha_{j s} w^{s 0}, \quad w^{j k}=\sum_{s=1}^{2} \alpha_{j s} w^{s k}, \quad j>2, \quad k=1,2 .
$$

Then the system $(33,36)$ reduces to the following 12 equations:

$$
\begin{aligned}
& L_{j 1} v-\left[B_{j}, v^{1}\right]=\left(L_{21} v-\left[B_{2}, v^{1}\right]\right) A^{j}, \quad j=3,4,5 \\
& L_{j 1} w^{k 0}-\sum_{i=1}^{2} \alpha_{j i} w^{k i}+w^{k 1} B_{j}=\left(L_{21} w^{k 0}-w^{k 2}+w^{k 1} B_{2}\right) A^{j}, j=3,4,5, k=0,1,2
\end{aligned}
$$

Following the previous procedure, we use equations (63) for $k=1$ to define the matrices $A^{j}$, and we substitute these definitions into equations (62) and (63) for $k=0$. Defining the blocks:

$$
\begin{aligned}
E_{j}^{v} & \equiv L_{j 1} v-\left[B_{j}, v^{1}\right], \quad j=2,3,4,5, \\
E_{j}^{w^{k 0}} & \equiv L_{j 1} w^{k 0}-\sum_{i=1}^{2} \alpha_{j i} w^{i k}+w^{1 k} B_{j}, \quad j=3,4,5, \quad k=0,1,2, \\
E_{2}^{w^{k 0}} & \equiv L_{21} w^{k 0}-w^{k 2}+w^{k 1} B_{2}, \quad k=0,1,2,
\end{aligned}
$$


we obtain:

$$
A^{j}=\left(E_{2}^{w_{10}}\right)^{-1} E_{j}^{w_{10}}, \quad j=3,4,5 .
$$

Substituting (65) into (62) and (63), we obtain the following closed system of 8 matrix PDEs for the 8 matrix fields $v, v_{1}, w_{00}, w_{10}, w_{20}, w_{11}, w_{12}, w_{22}$, consisting of the following 5 basic equations

$$
\begin{aligned}
E_{j}^{v}\left(E_{j}^{w^{10}}\right)^{-1} & =E_{2}^{v}\left(E_{2}^{w^{10}}\right)^{-1}, \quad j=3,4, \\
E_{j}^{w^{00}}\left(E_{j}^{w^{10}}\right)^{-1} & =E_{2}^{w^{00}}\left(E_{2}^{w^{10}}\right)^{-1}, \quad j=3,4,5,
\end{aligned}
$$

supplemented by two of the remaining equations (63), for instance, those for $j=3,4, k=2$ :

$$
E_{j}^{w^{20}}\left(E_{j}^{w^{10}}\right)^{-1}=E_{2}^{w^{20}}\left(E_{2}^{w^{10}}\right)^{-1}
$$

and by one (largely arbitrary) relation between the 8 matrix fields:

$$
F\left(v, v_{1}, w_{00}, w_{10}, w_{20}, w_{11}, w_{12}, w_{22}\right)=0
$$

which is introduced in the same spirit as before. Other equations of the system (62) and (63) can be treated as a symmetries of eqs. $(66,67)$.

This procedure can be generalized to an arbitrary number $n$ of dimensions, introducing the following equation for $G$ :

$$
G_{x^{j}}=\sum_{k=1}^{n-3} \alpha_{j k} G_{x^{k}}, \quad j>n-3,
$$

where $\alpha_{j k}$ are constant diagonal matrices and $n \geq 4$. The resulting nonlinear PDEs, possessing the same block structure as their lower dimensional analogies, will have dimensionality $n$ and, as we shall show in section 4.4, will be characterized by a manifold of analytic solutions of dimension $n-2$.

The possibility to increase the dimensionality of the PDEs and, at the same time, to increase proportionally the dimensionality of the manifold of solutions is due to the combined effect of the hypothesis: $\operatorname{dim} \operatorname{ker} \hat{\Psi}=1$ and of the introduction of the fields $w^{i j}$. Indeed, i) the property (25) implies the multidimensional linear problems (30) and, via equations $(51,65)$, the nontrivial mixing of the fields $v, v^{1}$ and $w^{i j}$; ii) the matrix fields $w^{i j}$ are associated with the outer dressing function $G$, whose dimensionality can be increased without obstacles (see the $(n-3)$-dimensional equation (69)), while the matrix fields $v, v^{1}$ are associated with the matrix function $C$, an ingredient of the classical dressing method, whose dimensionality is severely constrained (see the 1-dimensional equations (10)).

Note that, by construction, the derived systems possess higher symmetries: eqs. $(52,53)$ with $j \geq 5$ and eqs. $(66,67)$ with $j \geq 6$.

We end this section elaborating on the dimensionality of the space of analytic solutions constructed in this section. Consider, as an illustrative example, the system of equations (57),(59) in $n=4$ dimensions for the two matrix fields $w^{00}, w^{10}$, and interpret $x^{4}$ as time variable. One can view the first equation $E_{2}^{w^{00}}\left(E_{2}^{w^{10}}\right)^{-1}=E_{3}^{w^{00}}\left(E_{3}^{w^{10}}\right)^{-1}$ as defining, in principle, $w^{10}$ in terms of $w^{00}$ and its partial derivatives $w_{x^{j}}^{00}, j=1,2,3$. Replacing this relation into the second equation $E_{3}^{w^{00}}\left(E_{3}^{w^{10}}\right)^{-1}=E_{4}^{w^{00}}\left(E_{4}^{w^{10}}\right)^{-1}$, one obtains a single equation for $w^{00}$, depending linearly on $w_{x^{4}}^{00}$ and nonlinearly on the other derivatives. Since, as we shall see in Sec.4.4, our 
dressing algorithm generates analytic solutions depending on an arbitrary matrix function of $n-2=2$ variables, the space of analytic solutions of (57),(59) has dimension $n-2=2$. Similar arguments can be used for the systems (41), (44) and (66)-(68), which exhibit a single equation with first order derivative with respect to $t=x^{n}$. Slightly different is a system (52)(54), in which two equations involve first order $x^{4}$-derivatives of the two functions $v$ and $w^{00}$. Accordingly, its solution space depends on two arbitrary functions of two variables, see (114) of 4.4. Thus we have established that the space of analytic solutions of all the examples of this section is $(n-2)$-dimensional.

\subsection{Compatibility of linear spectral problems versus nonlinear PDEs}

It is well-known that integrable PDEs arise as the compatibility of overdetermined systems of linear problems for some eigenfunction $U(\lambda ; x)$. For instance, the N-wave system (18) in $2+1$ dimensions is the integrability condition for the Lax pair (15).

Such a picture is lost in our case, since there is no direct algebraic way to construct the nonlinear equations (32) as the compatibility condition of the linear systems (30). Indeed, the compatibility between equations (30) for $j \neq k$ leads to equation

$$
\begin{aligned}
& U\left(\left[B_{k}, L_{j 1} v-\left[B_{j}, v_{1}\right]\right]-\left[B_{j}, L_{k 1} v-\left[B_{k}, v_{1}\right]\right]\right)+\left(L_{21} U\right)\left(L_{k 1} A_{j}-L_{j 1} A_{k}\right)+ \\
& \left(L_{21} U\right)_{x^{1}}\left(A_{k} B_{j}-A_{j} B_{k}\right)+\left(L_{21} U\right)_{x^{k}} A_{j}-\left(L_{21} U\right)_{x^{j}} A_{k}=0,
\end{aligned}
$$

from which one cannot infer anything, since the terms $\left(L_{21} U\right)_{x^{j}}$ and $\left(L_{21} U\right)_{x^{k}}$ are not independent matrix functions of $\lambda$, being expressible in terms of $L_{21} U$ and $U$. But, to obtain such expressions, one has to use additional structure, i.e., the dressing equation $\hat{\Psi} L_{j 1} U=0$. Differentiating it with respect to $x^{k}$ and using equation (8), one obtains the homogeneous equation

$$
\hat{\Psi}\left[\left(L_{j 1} U\right)_{x^{k}}+U B_{k}\left(L_{j 1} v-\left[B_{j}, v_{1}\right]\right)\right]=0,
$$

which, due to (25), implies that

$$
\left(L_{j 1} U\right)_{x^{k}}=-U B_{k}\left(L_{j 1} v-\left[B_{j}, v_{1}\right]\right)+\left(L_{21} U\right) f^{j k}, \quad j \neq k,
$$

where $f^{n k}$ are functions of $x$ only. Substituting these relations (with $j=2$ ) in (70), one obtains:

$$
\begin{aligned}
& U\left(\left[B_{k}, E_{j}^{v}-E_{2}^{v} A_{j}\right]-\left[B_{j}, E_{k}^{v}-E_{2}^{v} A_{k}\right]\right)+ \\
& \left(L_{21} U\right)\left[L_{k 1} A_{j}-L_{j 1} A_{k}+f_{21}\left(A_{k} B_{j}-A_{j} B_{k}\right)+f_{2 k} A_{j}-f_{2 j} A_{k}\right]=0,
\end{aligned}
$$

and the independence of $U$ and $L_{21} U$ implies

$$
\begin{aligned}
& {\left[B_{k}, E_{j}^{v}-E_{2}^{v} A_{j}\right]=\left[B_{j}, E_{k}^{v}-E_{2}^{v} A_{k}\right],} \\
& L_{k 1} A_{j}-L_{j 1} A_{k}+f_{21}\left(A_{k} B_{j}-A_{j} B_{k}\right)+f_{2 k} A_{j}-f_{2 j} A_{k}=0 .
\end{aligned}
$$

We observe that equation (74a) does not imply directly the wanted equations (32). To obtain them, one should consider, instead, the compatibility between the linear problem (30) and equation (72), which leads to the following equation:

$$
U B_{k}\left(E_{j}^{v}-E_{2}^{v} A^{j}\right)+\left(L_{21} U\right)\left(A_{x^{k}}^{j}+f^{2 k} A^{j}-f^{j k}\right)=0 .
$$

Again the independence of $U$ and $L_{21} U$ implies the relations $f^{j k}=f^{2 k} A^{j}+A_{x^{k}}^{j}$, together with the wanted equations (32).

Summarizing, the linear 3-dimensional problems (30) contain only partial informations and their algebraic compatibility does not imply, alone, the nonlinear equations (32). 


\section{Solution space}

In the previous sections we have constructed partially integrable PDEs under the basic assumption that the integral equation (6) admits nontrivial homogeneous solutions. In this section we show i) how to choose the inner dressing functions in order to satisfy this assumption and, consequently, ii) how to construct the corresponding manifold of particular solutions of the partially integrable PDEs, expressed in terms of the dressing data $\Psi, \Phi, C$ and $G$. Note that, in our case, the novel dressing function $G$ appears, in comparison with the classical algorithm.

The solutions of equations (9) and (10) are:

$$
\begin{aligned}
\Phi(\lambda ; x) & =\int \Phi_{0}(\lambda, k) e^{k B \cdot x} d k \\
C(\mu ; x) & =\int e^{q B \cdot x} C_{0}(q, \mu) d q,
\end{aligned}
$$

where $k, q$ are scalar parameters and $\lambda, \mu$ are vector parameters of dimension $n-3$. Thus equations (8) yield:

$$
\Psi(\lambda, \mu ; x)=\int \Phi_{0}(\lambda, k) e^{(k+q) B \cdot x} C_{0}(q, \mu) \frac{d k d q}{k+q}+\Sigma(\lambda, \mu), \quad B_{1}=I .
$$

We remark that, in the case of $S$-integrable equations, the integration constant $\Sigma(\lambda, \mu)$ is chosen to be $\delta(\lambda-\mu)$. In our case, we need a special form for $\Sigma$ (see (82) below).

It is quite standard to assume that the measure $d \Omega(\lambda)$ have support on an open domain $\mathcal{D}$ of the $\lambda$-space, and on a disjoint discrete set of points $D=\left\{b_{1}, \ldots, b_{M}\right\}, D \cap \mathcal{D}=\emptyset$. Correspondingly, we use the following notation for the dressing functions.

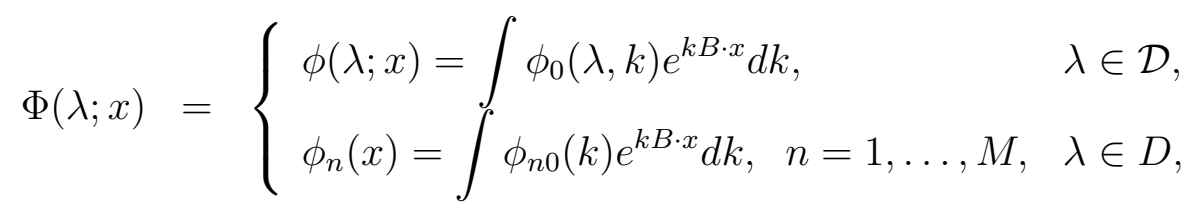

$$
\begin{aligned}
& C(\lambda ; x)= \begin{cases}c(\lambda ; x)=\int e^{q B \cdot x} c_{0}(q, \lambda) d q, & \lambda \in \mathcal{D}, \\
c_{n}(x)=\int e^{q B \cdot x} c_{n 0}(q) d q, & \lambda \in D, \quad n=1, \ldots, M,\end{cases} \\
& G(\lambda ; x)= \begin{cases}g(\lambda ; x), & \lambda \in \mathcal{D}, \\
g_{n}(x), & \lambda \in D, \quad n=1, \ldots, M\end{cases} \\
& U(\lambda ; x)= \begin{cases}u(\lambda ; x), & \lambda \in \mathcal{D}, \\
u_{n}(x), & \lambda \in D, \quad n=1, \ldots, M\end{cases}
\end{aligned}
$$

and we choose $\Sigma(\lambda, \mu)$ in the form:

$$
\Sigma(\lambda, \mu)= \begin{cases}\delta(\lambda-\mu), & \lambda, \mu \in \mathcal{D}, \\ \sigma_{n}(\lambda), & \lambda \in \mathcal{D} \mu \in D, \quad n=1, \ldots, M \\ \tilde{\sigma}_{n}(\mu), & \lambda \in D, \quad \mu \in \mathcal{D}, \quad n=1, \ldots, M \\ \sigma_{n m}, & \lambda, \mu \in D, \quad n, m=1, \ldots, M\end{cases}
$$


Then equation (6) reduces to the following system of $M+1$ equations

$$
\begin{aligned}
\phi(\lambda ; x)= & \sum_{j=1}^{M} \int \phi_{0}(\lambda, k) e^{(k+q) B \cdot x} c_{j 0}(q) \frac{d k d q}{k+q} u_{j}(x)+ \\
& \int_{\mathcal{D}} \phi_{0}(\lambda, k) e^{(k+q) B \cdot x} c_{0}(q, \mu) u(\mu ; x) \frac{d k d q d \Omega(\mu)}{k+q}+\sum_{j=1}^{M} \sigma_{j}(\lambda) u_{j}(x)+u(\lambda ; x), \quad \lambda \in \mathcal{D}, \\
\phi_{n}(x)= & \sum_{j=1}^{M} \int \phi_{n 0}(k) e^{(k+q) B \cdot x} c_{j 0}(q) \frac{d k d q}{k+q} u_{j}(x)+ \\
& \int_{\mathcal{D}} \phi_{n 0}(k) e^{(k+q) B \cdot x} c_{0}(q, \mu) u(\mu ; x) \frac{d k d q d \Omega(\mu)}{k+q}+ \\
& \sum_{j=1}^{M} \sigma_{n j} u_{j}(x)+\sum_{j=1}^{M} \int_{\mathcal{D}} \tilde{\sigma}_{n}(\mu) u(\mu ; x) d \Omega(\mu), \quad n=1, \ldots, M
\end{aligned}
$$

for the unknown matrix functions $u(\lambda ; x), \lambda \in \mathcal{D}$ and $u_{j}(x), j=1, \ldots, M$.

Once the solution is obtained, one constructs the matrix fields $v, v^{1}, w^{i j}$ using equations $(13,17,34)$ :

$$
\begin{aligned}
v(x) & =\int_{\mathcal{D}} c(\lambda ; x) u(\lambda ; x) d \Omega(\lambda)+\sum_{k=1}^{M} c_{k}(x) u_{k}(x), \\
v^{1}(x) & =\int_{\mathcal{D}}\left[\partial_{x_{1}} c(\lambda ; x)\right] u(\lambda ; x) d \Omega(\lambda)+\sum_{k=1}^{M}\left[\partial_{x_{1}} c_{k}(x)\right] u_{k}(x), \\
w^{00}(x) & =\int_{\mathcal{D}} g(\lambda ; x) u(\lambda ; x) d \Omega(\lambda)+\sum_{k=1}^{M} g_{k}(x) u_{k}(x), \\
w^{j 0}(x) & =\int_{\mathcal{D}}\left[\partial_{x_{j}} g(\lambda ; x)\right] u(\lambda ; x) d \Omega(\lambda)+\sum_{k=1}^{M}\left[\partial_{x_{j}} g_{k}(x)\right] u_{k}(x), \\
w^{i j}(x) & =\int_{\mathcal{D}}\left[\partial_{x_{i}} \partial_{x_{j}} g(\lambda ; x)\right] u(\lambda ; x) d \Omega(\lambda)+\sum_{k=1}^{M}\left[\partial_{x_{i}} \partial_{x_{j}} g_{k}(x)\right] u_{k}(x)
\end{aligned}
$$

\subsection{The condition $\operatorname{dim} \operatorname{ker} \hat{\Psi}=1$.}

Now we have to provide the condition $\operatorname{dim} \operatorname{ker} \hat{\Psi}=1$. We base our considerations on well known facts of the theory of linear integral operators. If the homogeneous equation

$$
\int \Psi(\lambda, \mu ; x) H(\mu ; x) d \Omega(\mu)=0
$$

has a nontrivial solution, then its adjoint equation

$$
\int \tilde{H}(\lambda ; x) \Psi(\lambda, \mu ; x) d \Omega(\lambda)=0
$$


has a nontrivial solution as well. If $\operatorname{dim} \operatorname{ker} \hat{\Psi}=1$, then the solution spaces of both equations (86) and (87) are one dimensional.

In our case, equation (87) reads

$$
\int \tilde{H}(\lambda ; x)\left[\int^{x^{1}} d x^{1^{\prime}} \Phi\left(\lambda ; x^{\prime}\right) C\left(\mu ; x^{\prime}\right)+\Sigma(\lambda, \mu)\right] d \Omega(\lambda), \quad x^{\prime}=\left.x\right|_{x^{1} \rightarrow x^{1^{\prime}}}
$$

In view of the independence of $\Phi$ and $\Sigma$, this equation is splitted into two equations:

$$
\int \tilde{H}(\lambda ; x) \Phi\left(\lambda ; x^{\prime}\right) d \Omega(\lambda)=\int \tilde{H}(\lambda ; x) \Sigma(\lambda, \mu) d \Omega(\lambda)=0,
$$

which have to be satisfied for all $x, x^{\prime}$ and $\mu$. This means that $\tilde{H}$ is independent of $x$ and, due to equation (76), the following two conditions must be satisfied:

$$
\begin{gathered}
\int \tilde{H}(\lambda) \Phi_{0}(\lambda, k) d \Omega(\lambda)=0, \quad \forall k \\
\int \tilde{H}(\lambda) \Sigma(\lambda, \mu) d \Omega(\lambda)=0, \quad \forall \mu
\end{gathered}
$$

for the existence of a nontrivial solution of the homogeneous equation (86).

It is important to remark that, at the same time, the condition (90) provides also the solvability of the inhomogeneous integral equation (6). Therefore no further constraint must be imposed.

We consider a particular way to satisfy conditions (90) and (91), choosing

$$
\tilde{H}(\lambda)= \begin{cases}0, & \lambda \in \mathcal{D} \\ \mathcal{A}_{j}, & \lambda \in D\end{cases}
$$

where the matrices $\mathcal{A}_{j}$ are constant and nonsingular, so that the conditions $(90,91)$ are constraints only for the discrete parts of $\Phi$ and $\Sigma$ :

$$
\sum_{j=1}^{M} \mathcal{A}_{j} \phi_{j}=\sum_{j=1}^{M} \mathcal{A}_{j} \tilde{\sigma}_{j}(\mu)=\sum_{j=1}^{M} \mathcal{A}_{j} \sigma_{j n}=0, \quad \mu \in \mathcal{D}, \quad n=1, \ldots, M .
$$

Due to (93), we have only $(M-1)$ independent equations in the system (84) and, consequently, the solutions $u_{j}(x)$ are constructed up to an arbitrary function $f(x)$.

We remark that terms containing $\sigma_{j}, \sigma_{i j}$ and $\tilde{\sigma}_{j}$ may disregarded in equations $(83,84)$. Indeed, the terms containing $\sigma_{j}$ in equation (83) can be incorporated in the first term of the RHS. Similarly, the terms with $\sigma_{i j}$ and $\tilde{\sigma}_{j}$ in the equation (84) can be incorporated in the first and second terms of this equation. Thus we set $\sigma_{j}=\tilde{\sigma}_{j}=\sigma_{i j}=0$ without loss of generality.

\subsection{Degenerate kernel.}

The system of linear equations (83,84), supplemented by the conditions (93), has a rich manifold of solutions. To construct explicit solutions, we choose, as usual, a degenerate kernel:

$$
c_{0}(q, \mu)=\sum_{j=1}^{\tilde{M}} \tilde{c}_{1 j}(q) \tilde{c}_{2 j}(\mu)
$$


In this case, equations $(83,84)$ reduce to the following linear system of $M+\tilde{M}$ equations

$$
\begin{aligned}
& \tilde{\phi}_{n}(x)=\sum_{j=1}^{M} \nu_{n j}(x) u_{j}(x)+\sum_{j=1}^{\tilde{M}} \tilde{\nu}_{n j}(x) \tilde{u}_{j}(x)+\tilde{u}_{n}(x), \quad n=1, \ldots, \tilde{M}, \\
& \phi_{n}(x)=\sum_{j=1}^{M} \rho_{n j}(x) u_{j}(x)+\sum_{j=1}^{\tilde{M}} \tilde{\rho}_{n j}(x) \tilde{u}_{j}(x), \quad n=1, \ldots, M,
\end{aligned}
$$

for the matrix fields $u_{j}(x), \tilde{u}_{k}(x), j=1, \ldots, M, k=1, \ldots, \tilde{M}$, where:

$$
\tilde{u}_{k}(x)=\int \tilde{c}_{2 k}(\lambda) u(\lambda ; x) d \Omega(\lambda)
$$

and where the given coefficients $\nu_{n j}, \tilde{\nu}_{n j}, \rho_{n j}, \tilde{\rho}_{n j}, \tilde{\phi}_{n}$ are defined in terms of the spectral functions:

$$
\begin{aligned}
& \nu_{n j}(x)=\int \tilde{\phi}_{n 0}(k) e^{(k+q) B \cdot x} c_{j 0}(q) \frac{d k d q}{k+q}, \quad \tilde{\nu}_{n j}(x)=\int \tilde{\phi}_{n 0}(k) e^{(k+q) B \cdot x} \tilde{c}_{1 j}(q) \frac{d k d q}{k+q}, \\
& \rho_{n j}(x)=\int \phi_{n 0}(k) e^{(k+q) B \cdot x} c_{j 0}(q) \frac{d k d q}{k+q}, \quad \tilde{\rho}_{n j}(x)=\int \phi_{n 0}(k) e^{(k+q) B \cdot x} \tilde{c}_{1 j}(q) \frac{d k d q}{k+q}, \\
& \tilde{\phi}_{n}(x)=\int \tilde{c}_{2 n}(\lambda) \phi(\lambda ; x) d \Omega(\lambda), \quad \tilde{\phi}_{n 0}(k)=\int_{\mathcal{D}} \tilde{c}_{2 n}(\lambda) \phi_{0}(\lambda, k) d \Omega(\lambda) .
\end{aligned}
$$

This algebraic system is obtained, as usual, applying the operator $\int_{\mathcal{D}} c_{2 n}(\mu ; x) d \Omega(\mu) \cdot$ to $(83)$.

Having constructed, from (95), (96), the $u_{s}(x)$ and the $\tilde{u}_{s}(x)$, one obtains the eigenfunction $u(\lambda ; x)$ via the formula:

$$
u(\lambda ; x)=\int \phi_{0}(\lambda, k) e^{k B \cdot x} d k-\sum_{j=1}^{M} \rho_{j}(\lambda ; x) u_{j}(x)-\sum_{j=1}^{\tilde{M}} \tilde{\rho}_{j}(\lambda ; x) \tilde{u}_{j}(x)
$$

where:

$$
\begin{aligned}
& \rho_{j}(\lambda ; x)=\int \phi_{0}(\lambda, k) e^{(k+q) B \cdot x} c_{j 0}(q) \frac{d k d q}{k+q} \\
& \tilde{\rho}_{j}(\lambda ; x)=\int \phi_{0}(\lambda, k) e^{(k+q) B \cdot x} \tilde{c}_{1 j}(q) \frac{d k d q}{k+q}
\end{aligned}
$$

At last, one constructs the matrix fields $v, v^{1}, w^{i j}$, solutions of the nonlinear PDEs of Sec. 3.2, from equations (85).

\subsection{Fixing the arbitrary function $f(x)$}

Due to the constraint (93), the solutions $u_{j}$ and $\tilde{u}_{j}$ of the algebraic system (95) depend linearly on an arbitrary matrix function $f(x)$. Then, via (85), also the fields $v, v^{1}, w^{i j}$ depend linearly on this arbitrary function. Such an arbitrary function is completely fixed by the largely arbitrary relation among the fields. 
To be more concrete, let us illustrate all these facts in the simplest case: $c_{0}(q, \mu)=0, M=2$. The constraint (93) implies that $\rho_{2 j}=-\mathcal{A}_{2}^{-1} \mathcal{A}_{1} \rho_{1 j}, j=1,2$; then, from the homogeneous version of $(96)$ :

$$
\begin{aligned}
& 0=\rho_{11}(x) H_{1}(x)+\rho_{12}(x) H_{2}(x), \\
& 0=\rho_{21}(x) H_{1}(x)+\rho_{22}(x) H_{2}(x),
\end{aligned}
$$

one verifies that the second equation is consequence of the first, while the first equation admits the solution

$$
H_{1}(x)=\rho_{11}^{-1}(x) f(x), \quad H_{2}(x)=-\rho_{12}^{-1}(x) f(x)
$$

depending linearly on the arbitrary matrix function $f(x)$ (compare with (26)). The general solution of the inhomogeneous algebraic system (96) is then given by:

$$
\begin{aligned}
& u_{1}(x)=\frac{1}{2} \rho_{11}^{-1}(x) \phi_{1}(x)+\rho_{11}^{-1}(x) f(x), \\
& u_{2}(x)=\frac{1}{2} \rho_{12}^{-1}(x) \phi_{1}(x)-\rho_{12}^{-1}(x) f(x),
\end{aligned}
$$

depending on the arbitrary matrix function $f(x)$ in a linear way as well (compare with $(27)$ ). Consequently, such a linear dependence on $f(x)$ will appear, via (85), also in the matrix fields $v, v^{1}, w^{i j}$.

We remark that one could always identify $f(x)$ with one of the $u_{j}$ 's, say, with $u_{1}(x)$, obtaining

$$
u_{1}(x)=f(x), \quad u_{2}(x)=\rho_{12}^{-1}(x)\left[\phi_{1}(x)-\rho_{11}(x) f(x)\right]
$$

this identification, which clearly leads to a less symmetric formula than (104), seems to become more convenient when $M>2$.

Now we show, always in the simplest case: $c_{0}(q, \mu)=0, M=2$, how the arbitrary function $f(x)$ gets fixed imposing the relation (37), which we choose in one of the forms pointed after equation (38).

Equations of Sec.3.2.1. For the equations of this section, choose $g(\lambda)=\delta(\lambda-a)$.

Then, using equation (85) for $w^{00}$, the constraint (39) becomes the linear equation

$$
\begin{aligned}
& \left.h_{0}(x)+\left[h_{1}(x) \rho_{11}^{-1}(x)+h_{2}(x) \rho_{12}^{-1}(x)\right)\right] \frac{\phi_{1}(x)}{2}+ \\
& \left.\left[h_{1}(x) \rho_{11}^{-1}(x)+h_{2}(x) \rho_{12}^{-1}(x)\right)\right] f(x)=\exp \left(\sum_{i=1}^{n} a_{i} x^{i}\right)
\end{aligned}
$$

for $f(x)$, where

$$
h_{j}(x)=-\int_{\mathcal{D}} g(\lambda ; x) \rho_{j}(\lambda ; x) d \Omega(\lambda)+g_{j}(x), \quad j=1,2, \quad h_{0}(x)=\int_{\mathcal{D}} g(\lambda ; x) \phi(\lambda ; x) d \Omega(\lambda)
$$

yielding the following explicit formula for $f$ :

$$
\begin{aligned}
& \left.f(x)=\left[h_{1}(x) \rho_{11}^{-1}(x)+h_{2}(x) \rho_{12}^{-1}(x)\right)\right]^{-1}\left[\exp \left(\sum_{i=1}^{n} a_{i} x^{i}\right)-\right. \\
& \left.\left.h_{0}(x)-\left[h_{1}(x) \rho_{11}^{-1}(x)+h_{2}(x) \rho_{12}^{-1}(x)\right)\right] \frac{\phi_{1}(x)}{2}\right],
\end{aligned}
$$

Analogously, using equation (85) for $v^{1}$, the constraint (42) becomes a linear equation for $f(x)$, whose explicit solution is

$$
\left.\left.f(x)=\left[h_{1}(x) \rho_{11}^{-1}(x)+h_{2}(x) \rho_{12}^{-1}(x)\right)\right]^{-1}\left[\gamma(x)-\left[h_{1}(x) \rho_{11}^{-1}(x)+h_{2}(x) \rho_{12}^{-1}(x)\right)\right] \frac{\phi_{1}(x)}{2}\right],
$$


where now:

$$
h_{j}(x)=\int q e^{q B \cdot x} c_{j 0}(q) d q, \quad j=1,2 .
$$

Equations of Sec.3.2.2. In this case, equation (69) implies that

$$
G(\lambda ; x)=\int_{\mathcal{D}} \exp \left[\sum_{j=1}^{n-3} \lambda_{j}^{\prime}\left(x^{j}+\sum_{k=n-2}^{n} \alpha_{k j} x^{k}\right)\right] G_{0}\left(\lambda^{\prime}, \lambda\right) d \Omega\left(\lambda^{\prime}\right) .
$$

If, in particular, $n=4$, as in equation (45), then the reduction $G=C$ is admissible, identifying $\alpha_{j}=\alpha_{j 1}=B_{j}$ and $G_{0}=C_{0}$ (see (77)), and one obtains the nonlinear PDEs (57), supplemented by the relation (68).

If this relation is an arbitrary linear relation between fields, it leads to a linear equation for $f(x)$. For instance, if we choose (59), then, using the equation (85) for $w^{11}$, the constraint becomes a linear equation for $f(x)$, whose explicit solution is

$$
\begin{aligned}
& \left.f(x)=\left[h_{1}(x) \rho_{11}^{-1}(x)+h_{2}(x) \rho_{12}^{-1}(x)\right)\right]^{-1}[\gamma(x)- \\
& \left.\left.\int_{\mathcal{D}} g_{x^{1} x^{1}}(\lambda ; x) \phi(\lambda ; x) d \Omega(\lambda)-\left[h_{1}(x) \rho_{11}^{-1}(x)+h_{2}(x) \rho_{12}^{-1}(x)\right)\right] \frac{\phi_{1}(x)}{2}\right],
\end{aligned}
$$

where:

$$
h_{j}(x)=-\int_{\mathcal{D}} g_{x^{1} x^{1}}(\lambda ; x) \rho_{j}(\lambda ; x) d \Omega(\lambda)+g_{x^{1} x^{1}}(x), \quad j=1,2 .
$$

In a similar way, one can treat more general relations in higher dimensions $n$.

\subsection{Dimensionality of the solution space}

The dimensionality of the space of analytic solutions generated by our dressing scheme is essentially defined by the dimensionality of two expressions

$$
\int C(\lambda ; x) \Phi(\lambda ; x) d \Omega(\lambda), \quad \int G(\lambda ; x) \Phi(\lambda ; x) d \Omega(\lambda) .
$$

The first term, involving the "inner" dressing functions $C(\lambda ; x)$ and $\Phi(\lambda ; x)$, appears also in the classical dressing; its dependence on the space-time coordinates is severely constrained and, consequently, it carries dimensionality 2 (see (24) and the considerations made there). The second term involves the "outer" dressing function $G(\lambda ; x)$, a novel feature of our dressing procedure; its dependence on the space-time coordinates is instead largely arbitrary, playing a crucial role in increasing the dimensionality of the solution space through the following novel mechanism.

As we have seen in Sec.4.3, from the largely arbitrary relation among the fields one construct $f(x)$ in terms of the spectral representations of the fields $v, v^{1}, w^{i j}$. If such relation involves the fields $w^{i j}$, whose spectral representations involve expressions like (114b), then the dimensionality of $f(x)$ is not severely constrained. Since the matrix fields $v, v^{1}, w^{i j}$, solutions of our PDEs, depend linearly on $f(x)$, their analytic solution space is not severely constrained too. Using this argument, it is possible to establish easily the dimensionality of the space of analytic solutions of our PDEs.

In Sec.3.2.1, the first term has higher dimensionality than the second, since $G$ does not depend on $x$; it follows that the dimensionality of the space of analytic solutions of the 4 dimensional PDEs constructed there is 2, like for integrable PDEs in $2+1$ dimensions. 
In Sec.3.2.2, the outer dressing function is (111) and the second term in equation (114) reads $\left(\right.$ choosing $\left.G_{0}\left(\lambda^{\prime}, \lambda\right)=\delta\left(\lambda^{\prime}-\lambda\right)\right)$ :

$$
\int_{\mathcal{D}} \exp \left[\sum_{j=1}^{n-3} \lambda_{j}\left(x^{j}+\sum_{k=n-2}^{n} \alpha_{k j} x^{k}\right)\right] \Phi_{0}(\lambda, k) \exp [k(B \cdot x)] d k d \Omega(\lambda) .
$$

Since $\lambda$ is a vector parameter of dimension $n-3$, and $k$ is a scalar parameter, the above expression has dimension $n-2$, being parameterized by the arbitrary function $\Phi_{0}(\lambda, k)$ of $n-2$ variables. Consequently, $n-2$ is the dimension of the constructed $f(x)$, and of all the fields appearing in the nonlinear PDEs.

Summarizing, the solutions we constructed depend on an arbitrary function of $n-2$ variables (114b) and on an arbitrary function of 2 variables (114a). Then, in the exceptional case $n=4$, the solutions depend on 2 arbitrary functions of 2 variables. This conclusion is valid for all the examples presented in the paper.

\section{Conclusions}

In this paper we have generalized the dressing method to construct systems of nonlinear PDEs in $n$ dimensions $(n>3)$ i) possessing a manifold of analytic solutions of dimension $n-2$ (a very large, but not complete, manifold), and ii) possessing higher symmetries. But the constructed PDEs do not seem to be the compatibility condition for overdetermined systems of linear PDEs, a characterizing feature of completely integrable systems in lower dimensions.

The above properties indicate that they are examples of partially integrable PDEs in multidimension possessing a very large, but not complete, space of analytic solutions.

A natural generalization of the algorithm presented in this paper consists in studying the case in which the integral operator $\hat{\Psi}$ of the dressing problem exhibits a higher dimensional kernel:

$$
\operatorname{dim} \operatorname{ker} \hat{\Psi}=D^{k e r}>1
$$

In this case, equation (30) is replaced by

$$
L_{m 1} U(\lambda ; x)+\sum_{n=2}^{D^{k e r}+1}\left(L_{n 1} U(\lambda ; x)\right) A^{m n}(x)=0, \quad m>D^{k e r}+1,
$$

and one needs $D^{k e r}$ conditions on $U$ to define the functions $A^{m n}$. The study of the structure of the associated partially integrable equations, and of the dimensionality of the associated analytic solution space is postponed to future investigations.

The authors thank the referees for useful comments. This work was supported by the INTAS Young Scientists Fellowship Nr. 04-83-2983, Grants RFBR 04-01-00508 and NSh 1716-2003.

\section{References}

[1] C.S.Gardner, J.M.Green, M.D.Kruskal, R.M.Miura, Phys.Rev.Lett, 19, 1095 (1967)

[2] V.E.Zakharov and A.B.Shabat, Funct.Anal.Appl., 8, 43 (1974) 
[3] V.E.Zakharov and A.B.Shabat, Funct.Anal.Appl., 13, 13 (1979)

[4] V.E.Zakharov and S.V.Manakov, Funct.Anal.Appl., 19, 11 (1985)

[5] L.V.Bogdanov and S.V.Manakov, J.Phys.A:Math.Gen., 21, L537 (1988)

[6] V. E. Zakharov, On the dressing method, in Inverse Methods in Action, edited by P. C. Sabatier, Springer-Verlag, Berlin, 602 (1990).

[7] V.E.Zakharov, S.V.Manakov, S.P.Novikov and L.P.Pitaevsky, Theory of Solitons. The Inverse Problem Method, Plenum Press (1984)

[8] B.Konopelchenko, Solitons in Multidimensions, World Scientific, Singapore (1993)

[9] R. Beals and R. R. Coifman, Scattering, transformations spectrales et equations d'evolution nonlineare, I and II at Seminaire Goulaoic-Meyer-Schwartz 1980-1981, exp. 22, and 1981-1982, exp. 21, Ecole Polytechnique, Palaiseau

[10] M. J. Ablowitz, D. BarYaacov and A. S. Fokas, Stud.Appl.Math., 69, 135 (1983)

[11] C. N. Yang and R. L. Mills, Phys.Rev, 96, 191 (1954)

[12] V. E. Zakharov, Integrable Systems in Multidimensional Spaces, Lecture Notes in Physics, 153, Springer-Verlag, Berlin, 190 (1982).

[13] V. E. Zakharov, Multidimensional Integrable Systems in Proceedings of the International Congress of Mathematicians, PWN Warsaw,1225 (1983).

[14] J. F. Plebanski, J.Math.Phys., 16, 2395 (1975).

[15] A.A.Belavin, A.M.Polyakov, A.S.Schwartz and Yu.S.Tyupkin, Phys.Lett.B, 59, 85 (1975)

[16] A.A.Belavin and V.E.Zakharov, Phys.Lett.B, 73, 53 (1978)

[17] M.F. Atiyah, V.G. Drinfeld, N.J. Hitchin, Yu. I. Manin, Phys.Lett.A, 65, 185 (1978)

[18] V.G. Drinfeld, Yu.I. Manin, Journal of Nuclear Phys., 29, 1646 (1979)

[19] L.V.Bogdanov and B.G.Konopelchenko, Phys.Lett.A, 345, 137 (2005)

[20] S.V.Manakov and P.M.Santini, Inverse scattering transform for vector fields and for the heavenly equation; arXiv:nlin.SI/0512043.

[21] M.J.Ablowitz and P.A.Clarkson, Solitons, Nonlinear Evolution Equations and Inverse Scattering, Cambridge University Press, Cambridge (1991)

[22] A.Zenchuk, J.Physics A:Math.Gen. 37, nn 25, 6557 (2004).

[23] F.Calogero in What is integrability by V.E.Zakharov, Springer, 1 (1990)

[24] P.M.Santini, M.J.Ablowitz and A.S.Fokas, J.Math.Phys., 25, 2614 (1984).

[25] D. J. Kaup, Physica D, 1, 45 (1980).

[26] A. S. Fokas, Phys.Rev.Lett., 51, 3 (1983).

[27] A. S. Fokas and M. J. Ablowitz, J.Math.Phys., 25, 2494 (1984). 